\title{
Cyclic Phosphatidic Acid inhibits the Secretion of Vascular Endothelial Growth Factor from Diabetic Human Coronary Artery Endothelial Cells through Peroxisome Proliferator-activated \\ Receptor Gamma
}

\section{Tamotsu Tsukahara $^{1^{*}}$, Ryoko Tsukahara ${ }^{2,3}$, Hisao Haniu ${ }^{4}$, Yoshikazu Matsuda ${ }^{5}$, and Kimiko Murakami-Murofushi ${ }^{3}$}

\footnotetext{
${ }^{1}$ Department of Molecular Pharmacology and Neuroscience, Nagasaki University Graduate School of Biomedical Sciences, 1-14 Bunkyo-machi, Nagasaki 852-8521, Japan

${ }^{2}$ Endowed Research Division of Human Welfare Sciences, Ochanomizu University, 2-1-1, Ohtsuka, Bunkyo-ku, Tokyo 112-8610, Japan

${ }^{3}$ Science and Education Center, Ochanomizu University, 2-1-1 Ohtsuka, Bunkyo-ku, Tokyo 112-861, Japan

${ }^{4}$ Department of Orthopedic Surgery, Shinshu University School of Medicine, 3-1-1 Asahi, Matsumoto, Nagano 390-8621, Japan

${ }^{5}$ Clinical Pharmacology Educational Center, Nihon Pharmaceutical University, Ina-machi, Saitama 362-0806, Japan
}

Running title: $c P A$ regulates AGP-elicited VEGF secretion

To whom correspondence should be addressed: Tamotsu Tsukahara, ${ }^{1}$ Department of Molecular Pharmacology and Neuroscience, Nagasaki University Graduate School of Biomedical Sciences, 1-14 Bunkyo-machi, Nagasaki 852-8521, Japan

E-mail: ttamotsu@nagasaki-u.ac.jp 
Keywords: Diabetic human coronary artery endothelial cells; cyclic phosphatidic acid; VEGF; peroxisome proliferator-activated receptor gamma 


\section{ABSTRACT}

Background: Cyclic phosphatidic acid (cPA) potently inhibits neointima formation by inhibiting PPAR $\gamma$. Results: cPA suppressed PPAR $\gamma$-mediated cell growth and migration in D-HCAECs.

Conclusion: cPA inhibited VEGF expression resulting in suppressing cell growth and migration in DHCAECs.

Significance: cPA plays a regulatory role of PPAR $\gamma$ in the vascular processes.

Atherosclerosis is a disease characterized by building up plaques formation and leads to a potentially serious condition in which arteries are clogged by fatty substances such as cholesterol. Increasing evidence suggests that atherosclerosis is accelerated in type II diabetes. Recent study reported that high level of alkyl glycerophosphate (AGP) was accumulated in atherosclerotic lesions. The presence of this phospholipid in mildly oxidized low-density lipoprotein (LDL) is likely to be involved in atherogenesis. It has been reported that the activation of peroxisome proliferator-activated receptor gamma plays a key role in developing atherosclerosis. Our previous result indicates that cyclic phosphatidic acid (cPA), one of bioactive lipids, potently suppresses neointima formation by inhibiting the activation of peroxisome proliferator-activated receptor gamma (PPAR $\gamma)$. However, the detailed mechanism is still unclear. In this study, to elucidate the mechanism of the cPA-PPAR $\gamma$ axis in the coronary artery endothelium, especially in patients with type II diabetes, we investigated the proliferation, migration, and secretion of VEGF in human coronary artery endothelial cells from diabetes patients (D-HCAECs). AGP induced cell growth and migration, however, cPA suppressed the AGP-elicited growth and migration in D-HCAECs. Moreover, AGP increased VEGF secretion from D-HCAECs, and this event was attenuated by cPA. Taken together, these results suggest that cPA suppresses VEGF-stimulated growth and migration in DHCAECs. These findings could be important for regulatory roles of PPAR $\gamma$ and VEGF in the vascular processes associated with diabetes and atherosclerosis. 


\section{INTRODUCTION}

Atherosclerosis is an inflammatory disease in which lesions or plaques build up in arteries [1] [2]. The first step in the development of atherosclerotic lesions is the activation of cells in blood-vessel walls, such as endothelial cells [3] [4]. It is well known that the endothelium is directly involved in vascular disease, stroke, heart disease, and diabetes [5]. Especially, diabetes has been reported to increase the risk of cardiovascular disease by accelerating the formation of atherosclerosis [6]. Diabetes-associated atherosclerosis and vascular diseases are the principal causes of morbidity and mortality in patients with diabetes [7].

Lysophosphatidic acid (LPA) is a bioactive lipid that is produced in serum after the activation of multiple biochemical pathways [8] [9]. Some clinical studies have reported that the plasma LPA level is correlated with vascular diseases and some species of LPA induce neointima formation, which is a characteristic feature of common vascular pathologies such as atherosclerosis. However, previous reports stated that neointima formation induced by unsaturated LPA is not mediated by LPA 1 and $\mathrm{LPA}_{2}$ receptors that are the major LPA-receptor subtypes of the G-protein coupled receptors (GPCRs) expressed in bloodvessel walls [10]. Thus numerous factors contribute to atherosclerosis, a complex disease that is accelerated by diabetes and metabolic syndrome. Moreover, because metabolic syndrome occurs commonly in people with type II diabetes which most of the increased incidence of cardiovascular diseases [11] [12].

We previously reported that unsaturated LPA species induced arterial-wall remodeling in rat and mouse non-injury model, and this response required peroxisome proliferator-activated receptor gamma (PPAR $\gamma)$ [13]. The function of PPAR $\gamma$ has been studied extensively, and a variety of synthetic and physiological agonists of this receptor have been identified [14], including thiazolidinediones (TZDs) and numerous natural ligands containing fatty acids and phospholipids [14]. Recently, selected phospholipid such as LPA and alkyl-glycerophosphate (AGP, also known as alkyl-LPA) were identified as agonists of 
PPAR $\gamma$ (Fig.1) [15] [16]. Later, we reported that AGP is a specific high-affinity and more potent agonist of PPAR $\gamma$ than LPA. Fujiwara and colleagues reported that AGP, an analog of LPA, is a weak agonist of LPA receptors except LPA 5 receptor, however, only LPA 5 receptor shows a preference for AGP than LPA [20]. Interestingly, AGP has a higher potency than LPA with respect to PPAR $\gamma$ activation [16]. Binding studies using the PPAR $\gamma$ ligand-binding domain showed that binding study of the AGP was similar to that of the TZD rosiglitazone [16]. We recently identified cyclic phosphatidic acid (cPA) is an endogenous PPAR $\gamma$ antagonist, and it is generated by phospholipase D2 (PLD2) [13]. These observations suggest that activation of PPAR $\gamma$ is likely to lead to a complex cellular response.

Earlier studies have shown that $\operatorname{PPAR} \gamma$, which plays a key role in the cardiovascular system, is expressed in all cell types of the blood-vessel wall and in monocytes and macrophages [21]. Coronary artery disease is the most common type of heart disease, and the leading cause of death from cardiovascular diseases is typically the result of atherosclerosis [22]. Our results thus raise the possibility of using cPA as a lead compound to develop new treatments that act on PPAR $\gamma$. However, the critical genes affected by the cPA-mediated inhibition in the endothelial cells of the coronary artery remain unknown. The migration of endothelial cells contributes to diverse aspects of vascular physiology such as the development of atherosclerosis. Currently, cPA plays a role in the anti-neointima activity of carotid arteries [13]; and, thus, it is important to understand the mechanisms that determine cPA sensitivity.

Here, we are focusing on the regulation of the vascular endothelial growth factor (VEGF) regulation induced by cPA. VEGF is a growth factor that was first identified to be specific to the vascular endothelium [23]. In mammals, the VEGF family consists of five members, VEGF-A, VEGF-B, VEGF-C, VEGF-D and the placenta growth factor (PLGF) [24]. VEGF is a potent mitogen and angiogenic growth factor that is the most critical regulator of vascular development [23]. Previously, VEGF was shown to play a role in endothelial cell proliferation, migration, and atherosclerosis [25]. Several studies have demonstrated that PPAR $\gamma$ agonists regulate the expression of various genes including VEGF and its receptors [26]. Rosiglitazone, one of TZDs and a synthetic agonist of PPAR $\gamma$, has been reported to suppresses VEGF expression through a PPAR $\gamma$-responsive element in the promoter of the VEGF gene, 
and pioglitazone, another synthetic agonist of PPAR $\gamma$, was reported to decrease VEGF expression [27]. On the contrary, other studies have reported that TZD drugs increase VEGF expression [26].

In diabetes, a key factor could be the potential involvement of VEGF in the development of atherosclerosis in patients with diabetes [28]. The discrepancies in the aforementioned results may have arisen from the use of distinct cell types, but the exact mechanisms remain unknown. Moreover, although cPA can arrest the growth of cells, including diabetic human coronary artery endothelial cells (DHCAECs), the relationship between the anti-cell growth effect of cPA and the regulation of VEGF expression is unknown. In this study, we determined that the PPAR $\gamma$ antagonist cPA potently inhibited the release of VEGF protein into the media which express high levels of PPAR $\gamma$ in D-HCAECs. The reduction in VEGF expression and secretion was linked to a decrease in the expression of the mRNA and the transcriptional inactivation of the VEGF promoter. We conclude that the inhibition of PPAR $\gamma$ downregulates VEGF expression and that this may be involved in the regulation of D-HCAECs proliferation and migration.

\section{EXPERIMENTAL PROCEDURES}

Materials - A rabbit polyclonal anti-PPAR $\gamma$ antibody (sc-7196), a mouse monoclonal anti- $\beta$-actin antibody (sc-47778), a PPAR $\gamma$ small interfering RNA (siRNA) (sc-38304), and a control siRNA (sc37007) were purchased from Santa Cruz Biotechnology Inc. (Santa Cruz, CA, USA). A mouse monoclonal VEGF antibody (11081) was purchased from Immuno-Biological Laboratories (Fujioka, Gunma, Japan). We obtained cPA (18:1) as a gift from Dr. Kimiko Murakami-Murofushi (Ochanomizu University, Tokyo, Japan). AGP (18:1) and LPA (18:1) were purchased from Cayman Chemical Company (Ann Arbor, MI, USA).

Cell cultures - Primary human coronary artery endothelial cells (HCAECs) and HCAECs from donors with type II diabetes (D-HCAECs) were purchased from Lonza (Walkersville, MD, USA) and propagated in endothelial cell growth medium (EBM ${ }^{\mathrm{TM}}-2$, Lonza) containing 5\% FBS and manufacturer- 
recommended supplemental growth factors (EGM-2 ${ }^{\mathrm{TM}}$ Bullekit $^{\mathrm{TM}}$ ), antibiotics, and antimycotics. All assays were performed on cells between passages 3 and 12 and were repeated at least 3 times in duplicate or triplicate.

Reporter gene assays - PPAR $\gamma$ activation was measured in HCAECs and D-HCAECs transfected with $125 \mathrm{ng}$ of the pGL3-PPRE-acyl-CoA oxidase luciferase vector, $62.5 \mathrm{ng}$ of the pcDNA3.1-PPAR $\gamma$ vector, and $12.5 \mathrm{ng}$ of the pSV- $\beta$-galactosidase (Promega) vector, which were constructed as previously reported [13,16]. At $24 \mathrm{~h}$ after transfection, cells were treated with Opti-MEM (Invitrogen) containing the test compounds dissolved in DMSO (up to $0.1 \%$ ) and cultured for an additional 20 h. Luciferase activity was measured using the ONE-Glo Luciferase Assay System (Promega) and a LuMate microplate luminometer (Awareness Technology, Inc., Palm City, FL, USA). To monitor the transcriptional regulation of VEGF promoter activity, we used the human VEGF gene promoter $(-2279$ to +54$)$ cloned into the luciferase reporter plasmid pGL2 (Promega). Plasmids were amplified in Escherichia coli DH5 $\alpha$ (Toyobo, Osaka, Japan) and isolated using the FastGene Plasmid Mini Kit (Nippon Genetics, Tokyo, Japan). HCAECs and D-HCAECs grown to $70 \%-80 \%$ confluence were transfected in 96 -well plates by using $0.5 \mu \mathrm{L}$ of Lipofectamine LTX (Life Technology) and 75 ng of plasmid DNA per well, according to the supplier's protocol. After $24 \mathrm{~h}$, the medium was changed and, where necessary, supplemented with AGP or cPA (1, 3 , or $10 \mu \mathrm{M})$. The cells were collected $24 \mathrm{~h}$ after incubation, lysed, and subjected to the luciferase assay.

Quantitative real-time PCR analysis - Total RNA was isolated from HCAECs and D-HCAECs by using a NucleoSpin ${ }^{\circledR}$ RNA II kit (TAKARA, Otsu, Japan), and $0.5 \mu$ g of the RNA was used for the subsequent synthesis of cDNA by using a ReverTra Ace qPCR RT Kit (Toyobo) as recommended by the manufacturer. The levels of mRNA were measured by using an ECO Real-Time PCR system (Illumina, Inc., San Diego, CA, USA) and SYBR Green Real-Time PCR Master Mix -Plus- (Toyobo) with the following primer pairs: PPAR $\gamma 1$, 5'-GTGGCCGCAGATTTGAAAGAAG-3' (Forward) and 5'TGTCAACCATGGTCATTTCG-3' (Reverse); PPAR 2 2, 5'-CAAACCCCTATTCCA TGCTGTT-3' (F) 
and 5'-AATGGCATCTCTGTGTCAACC-3' (R); LPA 1 , 5'-ACGGTCATTCCGTATGCAGC-3' (F) and 5'-GGATAGCCCCCATGACGAT-3' (R); LPA2, 5'-TCTTCCTGCTCATGGTGGC-3' (F) and 5'GCGTTGTTTCACGGTAGCG-3' (R); LPA L $^{\prime}$ 5'-TCCAACCTCATGGCCTTCTT-3' (F) and 5'GACCCACTCGTATGCGGAGA-3' (R); LPA $_{4}$, 5'-ATGCAACCACCACCTGCTTT-3' (F) and 5'AGGAATGATAAAACCAACAACTTCAA-3' (R); LPA5, 5'-CTCGGTGGTGAGCGTGTACATG-3' (F) and 5'-GCGTAGCGGTCCACGTTGAT-3' (R); LPA, 5'-GGCCAAGAGAGTCACAATTCAGG-3' (F) and 5'-CAGCGTCATTATGAGGTCTGC-3' (R); VEGF-A, 5'-AGGGCAGAATCATCACGAAGT3' (F) and 5'-AGGGTCTCGATTGGATGGCA-3' $\quad$ (R); $18 \mathrm{~S} \quad$ rRNA, 5'CAGCCACCCGAGATTGAGCA-3' (F) and 5'-TAGTAGCGACGGGCGGTGTG-3' (R). All PCRs were performed in $10 \mu \mathrm{L}$ mixtures in 48 -well PCR plates (Illumina). The cycling conditions were $95^{\circ} \mathrm{C}$ for $10 \mathrm{~min}$ (polymerase activation), followed by 40 cycles of $95^{\circ} \mathrm{C}$ for $15 \mathrm{~s}, 55^{\circ} \mathrm{C}$ for $15 \mathrm{~s}$, and $72^{\circ} \mathrm{C}$ for 30 s. To identify the housekeeping genes that were most suitable for normalizing the data, we initially used a human housekeeping gene primer set (TAKARA). After amplification, the samples were slowly heated from $55^{\circ} \mathrm{C}$ to $95^{\circ} \mathrm{C}$ and fluorescence was measured continuously to obtain a melting curve. The relative mRNA levels were quantified by using the formula $2^{-\Delta \Delta \mathrm{Cq}}$, where $\Delta \mathrm{Cq}$ is the difference between the threshold cycle of a target cDNA and an endogenous reference cDNA.

Small interfering RNAs - The expression of PPAR $\gamma$ and VEGF in HCAECs and D-HCAECs was inhibited by transfecting with siRNAs targeted to PPAR $\gamma$ and VEGF (Santa Cruz Biotechnology) by using Lipofectamine RNAiMAX (Invitrogen). Cells were cultured in 6-well plates (Iwaki, Tokyo, Japan) at a density of $5 \times 10^{4}$ cells/well in DMEM containing $10 \%$ FBS. Cells were transfected with 100 $\mathrm{pmol} / \mathrm{mL}$ of mRNA-specific siRNAs or a scrambled control siRNA. The reduction in PPAR $\gamma$ levels was confirmed using western blotting analysis.

Measurement of cell proliferation - HCAECs and D-HCAECs were seeded in 96-well culture plates $(5 \times$ $10^{3}$ cells/well), and cell proliferation was determined using the Cell Counting Kit-8 (Dojindo, Kumamoto, 
Japan). After cells were incubated for 24 h, $10 \mu \mathrm{L}$ of the Cell Counting Kit-8 solution was added to each well and the plates were incubated for $2 \mathrm{~h}$ in an incubator at $37^{\circ} \mathrm{C}$ with $5 \% \mathrm{CO}_{2}$. The amount of orange formazan dye was determined by measuring the absorbance at $450 \mathrm{~nm}$ in a microplate reader (Awareness Technology).

Wound-healing assay - Cell-migration assays were performed using $\mu$-Dish 35 mm culture inserts (Ibidi, Martinsried, Germany) according to the manufacturer's protocols. Briefly, D-HCAECs $\left(1.4 \times 10^{4}\right.$ cells/well) were seeded into each well of the culture inserts and incubated at $37^{\circ} \mathrm{C}$. After the cells were attached, the culture inserts were gently removed, and the cells were treated with 2\% FBS containing fresh medium supplemented with the vehicle, AGP, cPA, or the synthetic PPAR $\gamma$ antagonist T0070907. Immediately after removing the inserts $(0 \mathrm{~h})$ and at 3 and $6 \mathrm{~h}$, cultures were photographed using phasecontrast microscopy, and the widths of wounds were measured to calculate the mean percentage of closure under each culture condition.

Assessment of VEGF secretion by D-HCAECs and HCAECs in vitro - Cells were seeded in 60 mm dishes at a density of $5 \times 10^{4}$ cells/dish and incubated in 10\% FBS containing medium overnight, followed by incubation in serum-free medium for $24 \mathrm{~h}$. Levels of human VEGF in the samples were determined by performing a sandwich enzyme immunoassay by using a human VEGF Assay Kit-IBL (ImmunoBiological Laboratories, Fujioka, Japan).

Statistical analysis - Student's $t$ test was used for statistical comparisons and $p<0.05$ was considered statistically significant.

\section{RESULTS}

Humans express two PPAR $\gamma$ isoforms, $\operatorname{PPAR} \gamma_{1}$ and $\operatorname{PPAR} \gamma_{2}$ originated from alternative splicing [29]. We first examined the mRNA expression level of PPAR $\gamma_{1}$ and PPAR $\gamma_{2}$ in HCAECs and D-HCAECs. 
CPA regulates AGP-elicited VEGF secretion

As shown in Figure 2A, PPAR $\gamma_{1}$ mRNA was expressed at higher levels in D-HCAECs than in HCAECs. In contrast to PPAR $\gamma_{1}$, we did not detect the expression of PPAR $\gamma_{2}$ mRNA in the both cell types/lines. We next investigated PPAR $\gamma$ protein expression levels in the cells (Fig. 2B). We observed that the two primary cell lines expressed distinct levels of $\operatorname{PPAR} \gamma_{1}$, however, $\operatorname{PPAR} \gamma_{2}$ was hardly detectable. The expression levels of PPAR $\gamma_{1}$ and PPAR $\gamma_{2}$ proteins agreed well with those of the mRNAs expression.

However, in D-HCAECs, treatment with AGP and cPA did not alter PPAR $\gamma$ mRNA expression for $24 \mathrm{~h}$ (Fig. 3B). To test the function of the PPAR $\gamma$ receptor, we transfected the cells with a luciferase reporter plasmid. Interestingly, luciferase activity increased only in D-HCAECs after exposure to AGP (Fig.3C). Next, to establish that none of the six known LPA receptors $\left(\mathrm{LPA}_{1-6}\right)$ is required for activation of PPAR $\gamma$, we confirmed the expression levels of LPA ${ }_{1-6}$ mRNAs in HCAECs and D-HCAECs. As shown in Figure 3C, only $\mathrm{LPA}_{4}$ mRNA was highly expressed in the two cell lines, and the expression of this receptor in D-HCAECs was significantly greater than that in HCAECs. To examine the differences in $\mathrm{LPA}_{4}$ activation in response to cPA and AGP, we obtained full dose-response curves of the activation of $\mathrm{LPA}_{4}$ by cPA and AGP in D-HCAECs. Previous report suggested that cPA is a weak agonist of $\mathrm{LPA}_{4}$ and it has a significantly higher $\mathrm{EC}_{50}$ than LPA and AGP [30]. Furthermore, the endogenous PPAR $\gamma$ antagonist cPA inhibited the enhanced activation of PPAR $\gamma$ activation elicited by AGP. We found that these two cell types expresse high levels of $\mathrm{LPA}_{4}$ (Fig. 3D). For this reason, we also-tested the ligand properties of AGP (18:1) and cPA (18:1) on $\mathrm{LPA}_{4}$. As shown in Fig. 3D, although AGP 18:1 activated $\mathrm{LPA}_{4}$, cPA 18:1 was no response up to $10 \mu \mathrm{M}$ in $\mathrm{LPA}_{4}$ overexpressing cells. These results suggest that cPA might not be associated with $\mathrm{LPA}_{4}$ receptor in D-HCAEC cells.

We next investigated the effect of the growth properties of HCAECs. As shown in Figure 4A, DHCAECs obtained from diabetic donors grew faster than the HCAECs obtained from healthy donors; DHCAEC cell numbers were significantly higher than HCAEC cell after $24 \mathrm{~h}$ incubation using medium containing 5\% FBS and the manufacturer-recommend supplemental growth factors. We next investigated 
the effect of cPA on the growth in HCAECs. cPA did not affect on the growth in HCAECs (Fig.4B). however, cPA induced cell growth in a time-dependent manner in D-HCAECs (Fig.4C). D-HCAECs showed more responsive to cPA than HCAECs (Figs. 4B and 4C). These data suggest that the altered growth properties of the cells obtained from the diabetic donors may represent a requisite feature of the diabetic status. To evaluate how PPAR $\gamma$ affects the growth of D-HCAECs, PPAR $\gamma$ expression was knocked down using siRNAs (Fig.4F) (it should be 4D). PPAR $\gamma$-siRNA efficiently reduced the protein expression (Fig.4X). As shown in Figure 4D, and 4E, cell growth increased after transfection with PPAR $\gamma$-siRNA, an effect that was not observed using the control siRNA. These results demonstrate that cPA-mediated inhibition of cell growth is PPAR $\gamma$-dependent and indicate that D-HCAECs are more sensitive to cPA-induced growth inhibition than control HCAECs. To characterize the biological role of cPA-dependent inhibition of D-HCAEC growth, we determined the effect of the inhibition on the migration of D-HCAECs in a wound-healing assay. After the cells were attached, culture inserts were gently removed, and the cells were treated with fresh medium containing $2 \%$ FBS and photographed over the next $6 \mathrm{~h}$ to measure wound widths. Representative results are shown in Figure 5A, which demonstrate that D-HCAECs migrated markedly faster than HCAECs after a $6 \mathrm{~h}$ subculture. To determine whether cPA affects D-HCAEC migration, we cultured D-HCAECs in the presence of vehicle, AGP, cPA, or T0070907 and photographed the cells over the next 6 h to measure wound widths. Our results showed that AGP, but not cPA, strongly induced D-HCAEC migration (Fig. 5B). Furthermore, the synthetic PPAR $\gamma$ antagonist T0070907 strongly inhibited cell migration. To evaluate the effects of PPAR $\gamma$ on the migration of D-HCAECs, PPAR $\gamma$ expression was knocked down using siRNAs. As shown in Figure 5C, cell migration increased after transfection with the siRNA targeting PPAR $\gamma$ but not the control siRNA. These results suggest that PPAR $\gamma$ inhibition strongly influences D-HCAEC migration.

VEGF is a potent inducer of endothelial cell proliferation and migration [31]. Noteworthy, VEGF also induces expression of IL-6, and IL-8 in endothelial cell [32] [33]. We performed real-time PCR analysis to determine whether lysophospholipids PPAR $\gamma$ ligands affects the levels of VEGF mRNA in vitro. As shown in Fig. 6A, VEGF mRNA levels in D-HCAECs were markedly enhanced after treatment 
with $1 \mu \mathrm{M}$ AGP and further increased when $10 \mu \mathrm{M}$ AGP was added; these are the concentrations of AGP that are present physiologically [16]. Furthermore, LPA also increased VEGF mRNA levels in DHCAECs in a dose-dependent manner (Fig. 6A). Interestingly, real-time PCR analysis revealed that, in DHCAECs, cPA decreased VEGF mRNA expression (Fig.6A). To determine how VEGF secretion is stimulated, HCAECs and D-HCAECs were incubated in the absence or presence of 1,3 , and $10 \mu \mathrm{M}$ AGP and then conditioned media were assayed for secreted VEGF by performing ELISAs. As shown in Fig. 6B, AGP were significantly increased VEGF secretion dose-dependently in D-HCAECs.

Representative results are shown in Figure 6C, which demonstrate that AGP and LPA but not cPA strongly induced VEGF secretion by D-HCAECs; cPA at concentrations as high as 1, 3, and $10 \mu \mathrm{M}$ failed to induce VEGF secretion, whereas AGP and LPA increased VEGF secretion by D-HCAECs in a dosedependent manner (Fig. 6C). These data suggest that exogenously applied cPA inhibited VEGF secretion elicited by LPA or AGP. Next, we determined effect of PPAR $\gamma$ ligands on the activity of the VEGF gene promoter (Fig. 6D). The cells were transfected with a reporter plasmid containing the VEGF promoter and then incubated with AGP or cPA. In D-HCAECs, the promoter activity was substantially enhanced compared with that in HCAECs after treatment with AGP but not cPA. Intriguingly, cPA inhibited the VEGF-promoter activation induced by AGP.

These data suggest that the effect of the PPAR $\gamma$ antagonist cPA on VEGF mRNA expression is mediated at least partly by a decrease in the rate of transcription from the VEGF promoter. We also explored the influence of VEGF per se on the growth of D-HCAECs. As shown in Figure 6E, cPA inhibited exogenously added VEGF enhanced D-HCAEC growth. Furthermore, transfection of DHCAECs with control or VEGF siRNA revealed that the VEGF knockdown (Fig.7A and B) attenuated AGP-induced VEGF secretion (Fig. 7C) and cell growth promotion (Fig. 7D). Furthermore, as shown in Fig.7E, the level of IL-6, and IL-8 were significantly elevated in the AGP treated co-culture medium of D-HCAEC. 


\section{DISCUSSION}

Diabetes patients have been reported to have a 2- to 4-fold increased risk of developing atherosclerotic coronary artery disease [34]. Endothelial dysfunction has been suggested to be an early event in diabetic atherosclerosis, and this dysfunction is associated with atherosclerotic coronary artery disease risk [35]. Brief exposure to AGP has been reported to induce progressive neointima formation [10] and, recently, LPA and its analog were shown to stimulate a new type of signaling by directly activating PPAR $\gamma$ [15]. A previous report suggested that neointima formation elicited by unsaturated LPA is not mediated by the LPA receptors (GPCRs) expressed in blood-vessel walls [13]; however, topical application of unsaturated LPA species into the non-injured carotid artery of rodents induced arterial-wall remodeling [13], and this response required PPAR $\gamma$. LPA and AGP have also been reported to be agonists of PPAR $\gamma$ and have been implicated in atherogenesis [10]. When AGP was infused to an injured carotid artery, neointimal thickening was augmented, whereas the TZD drug rosiglitazone attenuated neointimal thickening induced by mechanical injury. However, in a non-injury model, rosiglitazone induced neointima formation when applied intraluminally into the carotid artery [13]. Thus, AGP, but not rosiglitazone, increased injury-induced neointima formation, indicating that AGP-mediated PPAR $\gamma$ activation plays a role in vascular remodeling following vascular injury [13].

PPAR $\gamma$ is a member of the nuclear receptor gene family that plays a central role in the regulation of glucose and lipid homeostasis [36]. PPAR $\gamma$ plays a critical role in the cardiovascular system, and it is expressed in all cell types of the vessel wall, as well as in monocytes and macrophages [37]. PPAR $\gamma$ agonists promote adipocytic differentiation and stimulate the uptake of low-density lipoprotein by macrophages, leading to foam-cell formation in the arterial wall [38]. Considerable evidence suggests that oxidized phospholipids and fatty acids function as deleterious signaling molecules in the context of atherosclerosis lesion formation [39]. We recently determined that the production of cPA inhibits the transcription of PPAR $\gamma$-target genes that normally drive adipocytic differentiation, lipid accumulation in macrophages, and arterial-wall remodeling; our recent work suggests that the endogenous LPA analog 
cPA is a bona-fide second messenger and a physiological inhibitor of PPAR $\gamma$ [13]. However, the specific effects of diabetes on the function of the coronary endothelium are not well defined.

In this study, we determined that the growth and migration of human coronary endothelial cells obtained from donors with type II diabetes and the suppressive effects of the phospholipid cPA require the inhibition of PPAR $\gamma$ (Fig.8). Furthermore, our results have demonstrated that, in HCAECs isolated from diabetes patients, which expressed PPAR $\gamma$ at high levels, VEGF production was elicited by AGP. We have shown that treatment with the PPAR $\gamma$ antagonist cPA diminished the synthesis of VEGF, which suggests that cPA inhibition of VEGF synthesis can affect the vessel wall by influencing endothelial cell growth and migration. Conversely, AGP, the high-affinity natural ligand of PPAR $\gamma$ [16], effectively increased VEGF mRNA expression and protein release. The VEGF promoter, however, does not contain the PPRE motif; thus, cPA appears to downregulate VEGF expression indirectly, and the reduction in the synthesis of mRNA and protein probably depends partly on the inhibition of the VEGF promoter. Several studies have reported increased levels of VEGF in the serum of patients with acute coronary syndrome [40]. Atherosclerosis is the most common type of heart disease and the leading cause of death resulting from cardiovascular diseases. In addition, previous report suggests that VEGF appears to play a central role in mediating diabetic endothelial dysfunction. [41]. Thus, the results of this study raise the possibility of using cPA as a lead compound to develop new treatments that act on PPAR $\gamma$; however, further investigation is required to determine the molecular mechanisms underlying the described effect of cPA. 


\section{REFERENCES}

1. Hansson GK, Robertson AK, Soderberg-Naucler C (2006) Inflammation and atherosclerosis. Annu Rev Pathol 1: 297-329.

2. Libby P (2006) Inflammation and cardiovascular disease mechanisms. Am J Clin Nutr 83: 456S-460S.

3. Sumpio BE, Riley JT, Dardik A (2002) Cells in focus: endothelial cell. Int J Biochem Cell Biol 34: 1508-1512.

4. Davignon J, Ganz P (2004) Role of endothelial dysfunction in atherosclerosis. Circulation 109: III2732.

5. Rajendran P, Rengarajan T, Thangavel J, Nishigaki Y, Sakthisekaran D, et al. (2013) The Vascular Endothelium and Human Diseases. Int J Biol Sci 9: 1057-1069.

6. Kannel WB, McGee DL (1979) Diabetes and cardiovascular disease. The Framingham study. JAMA 241: 2035-2038.

7. Cade WT (2008) Diabetes-related microvascular and macrovascular diseases in the physical therapy setting. Phys Ther 88: 1322-1335.

8. Moolenaar WH (1995) Lysophosphatidic acid, a multifunctional phospholipid messenger. J Biol Chem 270: $12949-12952$.

9. Tigyi G, Parrill AL (2003) Molecular mechanisms of lysophosphatidic acid action. Prog Lipid Res 42: 498-526.

10. Zhang C, Baker DL, Yasuda S, Makarova N, Balazs L, et al. (2004) Lysophosphatidic acid induces neointima formation through PPARgamma activation. J Exp Med 199: 763-774.

11. Grundy SM, Benjamin IJ, Burke GL, Chait A, Eckel RH, et al. (1999) Diabetes and cardiovascular disease: a statement for healthcare professionals from the American Heart Association. Circulation 100: 1134-1146.

12. Lau DC, Yan H, Dhillon B (2006) Metabolic syndrome: a marker of patients at high cardiovascular risk. Can J Cardiol 22 Suppl B: 85B-90B. 
13. Tsukahara T, Tsukahara R, Fujiwara Y, Yue J, Cheng Y, et al. (2010) Phospholipase D2-dependent inhibition of the nuclear hormone receptor PPARgamma by cyclic phosphatidic acid. Mol Cell 39: 421-432.

14. Tsukahara T (2012) The Role of PPARgamma in the Transcriptional Control by Agonists and Antagonists. PPAR Res 2012: 362361.

15. McIntyre TM, Pontsler AV, Silva AR, St Hilaire A, Xu Y, et al. (2003) Identification of an intracellular receptor for lysophosphatidic acid (LPA): LPA is a transcellular PPARgamma agonist. Proc Natl Acad Sci U S A 100: 131-136.

16. Tsukahara T, Tsukahara R, Yasuda S, Makarova N, Valentine WJ, et al. (2006) Different residues mediate recognition of 1-O-oleyllysophosphatidic acid and rosiglitazone in the ligand binding domain of peroxisome proliferator-activated receptor gamma. J Biol Chem 281: 3398-3407.

17. Sugiura T, Nakane S, Kishimoto S, Waku K, Yoshioka Y, et al. (1999) Occurrence of lysophosphatidic acid and its alkyl ether-linked analog in rat brain and comparison of their biological activities toward cultured neural cells. Biochim Biophys Acta 1440: 194-204.

18. Sugiura T, Nakane S, Kishimoto S, Waku K, Yoshioka Y, et al. (2002) Lysophosphatidic acid, a growth factor-like lipid, in the saliva. J Lipid Res 43: 2049-2055.

19. Nakane S, Tokumura A, Waku K, Sugiura T (2001) Hen egg yolk and white contain high amounts of lysophosphatidic acids, growth factor-like lipids: distinct molecular species compositions. Lipids 36: 413-419.

20. Williams JR, Khandoga AL, Goyal P, Fells JI, Perygin DH, et al. (2009) Unique ligand selectivity of the GPR92/LPA5 lysophosphatidate receptor indicates role in human platelet activation. J Biol Chem 284: 17304-17319.

21. Bishop-Bailey D (2000) Peroxisome proliferator-activated receptors in the cardiovascular system. Br J Pharmacol 129: 823-834.

22. Roger VL, Go AS, Lloyd-Jones DM, Benjamin EJ, Berry JD, et al. (2012) Heart disease and stroke statistics--2012 update: a report from the American Heart Association. Circulation 125: e2-e220. 
23. Ferrara N, Gerber HP, LeCouter J (2003) The biology of VEGF and its receptors. Nat Med 9: 669-676.

24. Olsson AK, Dimberg A, Kreuger J, Claesson-Welsh L (2006) VEGF receptor signalling - in control of vascular function. Nat Rev Mol Cell Biol 7: 359-371.

25. Inoue M, Itoh H, Ueda M, Naruko T, Kojima A, et al. (1998) Vascular endothelial growth factor (VEGF) expression in human coronary atherosclerotic lesions: possible pathophysiological significance of VEGF in progression of atherosclerosis. Circulation 98: 2108-2116.

26. Yoshizaki T, Motomura W, Tanno S, Kumei S, Yoshizaki Y, et al. (2010) Thiazolidinediones enhance vascular endothelial growth factor expression and induce cell growth inhibition in nonsmall-cell lung cancer cells. J Exp Clin Cancer Res 29: 22.

27. Peeters LL, Vigne JL, Tee MK, Zhao D, Waite LL, et al. (2005) PPAR gamma represses VEGF expression in human endometrial cells: implications for uterine angiogenesis. Angiogenesis 8: 373-379.

28. Celletti FL, Waugh JM, Amabile PG, Brendolan A, Hilfiker PR, et al. (2001) Vascular endothelial growth factor enhances atherosclerotic plaque progression. Nat Med 7: 425-429.

29. Mukherjee R, Jow L, Croston GE, Paterniti JR, Jr. (1997) Identification, characterization, and tissue distribution of human peroxisome proliferator-activated receptor (PPAR) isoforms PPARgamma2 versus PPARgamma1 and activation with retinoid X receptor agonists and antagonists. J Biol Chem 272: 8071-8076.

30. Baker DL, Fujiwara Y, Pigg KR, Tsukahara R, Kobayashi S, et al. (2006) Carba analogs of cyclic phosphatidic acid are selective inhibitors of autotaxin and cancer cell invasion and metastasis. J Biol Chem 281: 22786-22793.

31. Unemori EN, Ferrara N, Bauer EA, Amento EP (1992) Vascular endothelial growth factor induces interstitial collagenase expression in human endothelial cells. J Cell Physiol 153: 557-562.

32. Cohen T, Nahari D, Cerem LW, Neufeld G, Levi BZ (1996) Interleukin 6 induces the expression of vascular endothelial growth factor. J Biol Chem 271: 736-741. 
33. Martin D, Galisteo R, Gutkind JS (2009) CXCL8/IL8 stimulates vascular endothelial growth factor (VEGF) expression and the autocrine activation of VEGFR2 in endothelial cells by activating NFkappaB through the CBM (Carma3/Bcl10/Malt1) complex. J Biol Chem 284: 6038-6042.

34. Luscher TF, Creager MA, Beckman JA, Cosentino F (2003) Diabetes and vascular disease:

pathophysiology, clinical consequences, and medical therapy: Part II. Circulation 108: 1655-1661.

35. Hadi HA, Carr CS, Al Suwaidi J (2005) Endothelial dysfunction: cardiovascular risk factors, therapy, and outcome. Vasc Health Risk Manag 1: 183-198.

36. Spiegelman BM (1998) PPAR-gamma: adipogenic regulator and thiazolidinedione receptor. Diabetes 47: 507-514.

37. Duan SZ, Usher MG, Mortensen RM (2008) Peroxisome proliferator-activated receptor-gammamediated effects in the vasculature. Circ Res 102: 283-294.

38. Cock TA, Houten SM, Auwerx J (2004) Peroxisome proliferator-activated receptor-gamma: too much of a good thing causes harm. EMBO Rep 5: 142-147.

39. Bochkov VN, Oskolkova OV, Birukov KG, Levonen AL, Binder CJ, et al. (2010) Generation and biological activities of oxidized phospholipids. Antioxid Redox Signal 12: 1009-1059.

40. Konopka A, Janas J, Piotrowski W, Stepinska J (2013) Concentration of vascular endothelial growth factor in patients with acute coronary syndrome. Cytokine 61: 664-669.

41. de Vriese AS, Tilton RG, Elger M, Stephan CC, Kriz W, et al. (2001) Antibodies against vascular endothelial growth factor improve early renal dysfunction in experimental diabetes. J Am Soc Nephrol 12: 993-1000.

\section{ACKOWLEDGMENTS}

This work was supported by Grants-in-Aid for Scientific Research (B) (25293274 to Tamotsu Tsukahara) from the Japan Society for the Promotion of Science (JSPS) KAKENHI. 


\section{FIGURE LEGENDS}

Figure 1. Chemical structures of LPA, AGP, and cPA. It is made up of a glycerol backbone with a hydroxyl group, a phosphate group, and a long-chain saturated or unsaturated fatty acid. AGP is an alkylether analog of LPA. AGP shows a higher potency than LPA for the intracellular LPA receptor PPAR $\gamma$. cPA is a naturally occurring acyl analog of LPA. cPA is a weak agonist of plasma membrane LPA receptors, whereas cPA is an inhibitor of PPAR $\gamma$.

Figure 2. Comparison of endogenous PPAR $\gamma$ expression in primary human coronary artery endothelial cells (HCAECs; from healthy donors) and D-HCAECs (from donors with type II diabetes). (A) Real-time PCR measurement of PPAR $\gamma_{1}$ and PPAR $\gamma_{2}$ mRNA expression in the tested cell lines. The PPAR $\gamma_{1}$ and PPAR $\gamma_{2}$ levels were normalized relative to $\beta$-actin and are expressed as mean \pm SEM $(n=3),{ }^{* *} p<0.01$. (B) Representative western blots showing the expression of PPAR $\gamma_{1}$ and PPAR $\gamma_{2}$ in HCAECs and DHCAECs from different donors. PPAR $\gamma$ proteins were separated by performing SDS-PAGE and then the levels were compared by visualizing immunoreactive bands by using an enhanced chemiluminescence reagent. Each lane was loaded with $10 \mu \mathrm{g}$ of whole-cell lysate, and $\beta$-actin was used as the loading control.

Figure 3. (A) Real-time PCR measurement of LPA $1-6$ mRNA expression in the tested cell lines. Total RNA was extracted from the cells and the expression level of each LPA receptor was determined using quantitative real-time PCR. The relative levels of $\mathrm{LPA}_{1-6}$ normalized to $\beta$-actin are expressed as mean \pm $\operatorname{SEM}(\mathrm{n}=3),{ }^{* *} p<0.01$. (B) Real-time PCR measurement of the expression of PPAR $\gamma_{1}$ mRNA in HCAECs and D-HCAECs. Cells were incubated with $10 \mu \mathrm{M}$ LPA (18:1) or cPA (18:1) for $24 \mathrm{~h}$. The relative PPAR $\gamma_{1}$ mRNA levels, normalized to $18 \mathrm{~S}$ rRNA, are expressed as mean $\pm \mathrm{SEM}(\mathrm{n}=3),{ }^{* *} p<$ 0.01. (C) HCAECs and D-HCAECs were transfected with the PPRE-luc and CMV- $\beta$-galactosidase plasmids for $72 \mathrm{~h}$ and then treated with vehicle or the indicated PPAR $\gamma$ ligands for $24 \mathrm{~h}$. The level of luciferase activity was measured in lysates of the treated cells and normalized to $\beta$-galactosidase activity. Data are presented as mean \pm SEM $(n=5),{ }^{* *} p<0.01$. (D) Transfection of $\mathrm{LPA}_{4}$ into D-HCAECs results 
in LPA- and cPA-elicited $\mathrm{Ca}^{2+}$ transients. $\mathrm{LPA}_{4}$ transfected D-HCAECs and WT do not respond to cPA (up to $10 \mu \mathrm{M}$ ). Real-time PCR measurement of the overexpression of $\mathrm{LPA}_{4}$ in D-HCAECs (Right figure). Data are presented as mean $\pm \operatorname{SEM}(\mathrm{n}=4),{ }^{* *} p<0.01$

Figure 4. Effects of cPA on the proliferation of HCAECs and D-HCAECs under $0.1 \%$ BSA (fatty acid free ) medium. Time-dependent cell proliferation was measured using the Cell Counting Kit-8 at 0, 12, 24, and $48 \mathrm{~h}$ after incubation in EBSS medium alone (A) or in medium containing $10 \mu \mathrm{M} \mathrm{cPA}$ (B and C). Equal number of cells $\left(1 \times 10^{4}\right.$ cells/well $)$ were seeded in 96 -well plates and cultured overnight at $37^{\circ} \mathrm{C}$ in an incubator with $5 \% \mathrm{CO}_{2}$. Subsequently, compounds were added to the medium, and cells were incubated for the indicated times. (D) Expression of PPAR $\gamma$ was knocked down in HCAECs and DHCAECs by using siRNAs. Time-dependent inhibition of cell growth was measured using the Cell Counting Kit-8 at 0, 12, 24, 48, and 72 h after siRNA transfection. Equal number of cells $\left(1 \times 10^{4}\right.$ cells/well) were seeded in 96 -well plates and cultured for $24 \mathrm{~h}$ at $37^{\circ} \mathrm{C}$ in an incubator with $5 \% \mathrm{CO}_{2}$. Next, $10 \mu \mathrm{L}$ of the Cell Counting Kit-8 reagent was added to the medium and the cells were maintained for $2 \mathrm{~h}$ in the incubator (with $5 \% \mathrm{CO}_{2}$ ). The amount of orange formazan dye generated was determined by measuring the absorbance at $450 \mathrm{~nm}$ in a microplate reader. Data are expressed as mean \pm SEM $(\mathrm{n}=3)$, ${ }^{* *} p<0.01$.

Figure 5. Effect of cPA on the migration of HCAECs and D-HCAECs. (A) Migration of HCAECs and D-HCAECs was measured using a wound-healing assay. Images were captured after incubation for 0, 3, and $6 \mathrm{~h}$ by using an Olympus CKX31 inverted microscope (40 × magnification) equipped with a digital camera (MDCE-5B2, Shodensha, Osaka, Japan). (B) The migration of HCAECs and D-HCAECs coincubated with $10 \mu \mathrm{M}$ LPA, cPA, and T0070907 was measured using a wound-healing assay. Images were captured after incubation for 0, 3, and $6 \mathrm{~h}$ as described in (A). Data from the wound-healing assays are presented as mean $\pm \operatorname{SEM}(\mathrm{n}=3),{ }^{* *} p<0.01$. Data are expressed as percentages of wound closure relative to the wound width at Time 0 . Wound closure in untreated cells was regarded as $100 \%$. (C) D- 
HCAEC cell migration increased after transfection with the siRNA targeting PPAR $\gamma$ but not the control siRNA.

Figure 6. Real-time PCR measurement of VEGF mRNA expression in HCAECs and D-HCAECs. (A) HCAECs and D-HCAECs were treated with $10 \mu \mathrm{M}$ cPA, LPA, or AGP for $20 \mathrm{~h}$. The relative VEGF levels normalized to $18 \mathrm{~S}$ rRNA are expressed as mean $\pm \operatorname{SEM}(\mathrm{n}=3),{ }^{* *} p<0.01$. (B) D-HCAECs were treated with indicated concentrations of cPA (18:1), AGP, or LPA (18:1) for $20 \mathrm{~h}$. PCR was performed using specific primers for PSF. The relative VEGF levels normalized to 18S rRNA are expressed as mean \pm SEM ( $\mathrm{n}=3),{ }^{* *} p<0.01$. (C) VEGF secretion in response to in vitro treatment with AGP. HCAECs and D-HCAECs were treated with AGP for $24 \mathrm{~h}$, and VEGF secretion was assessed by performing ELISAs. Data from the VEGF assays are presented as mean \pm SEM $(n=3),{ }^{* *} p<0.01$. LPA but not cPA dosedependently increased VEGF release from D-HCAECs. The culture supernatants were collected after incubation for $24 \mathrm{~h}$ with DMSO (vehicle), LPA (18:1), AGP (1, 3, and $10 \mu \mathrm{M})$, or cPA (18:1) (1, 3, and $10 \mu \mathrm{M}$ ). The VEGF levels in the supernatant were analyzed by performing ELISAs. (D) The effect of PPAR $\gamma$ ligands on the activity of the VEGF promoter, measured by performing luciferase assays on transiently transfected HCAECs and D-HCAECs. (E) cPA inhibited exogenously added VEGF enhanced D-HCAEC cell growth. D-HCAEC cells. An equal number of cells $\left(1 \times 10^{4}\right.$ cells/well $)$ were seeded in 96 well plates and cultured overnight at $37^{\circ} \mathrm{C}$ in an incubator with $5 \% \mathrm{CO}_{2}$. Subsequently, VEGF $(50 \mathrm{ng} / \mathrm{mL})$ and cPA $(10 \mu \mathrm{M})$ were added to the medium, and the cells were incubated for $12 \mathrm{~h}$. The amount of orange formazan dye generated was determined by measuring the absorbance at $450 \mathrm{~nm}$ in a microplate reader. Data are expressed as mean $\pm \operatorname{SEM}(\mathrm{n}=3),{ }^{* *} p<0.01$.

Figure 7. Down-regulation of VEGF inhibits the proliferation of D-HCAECs. (A) Expression of VEGF was knocked down in D-HCAECs. Total protein was extracted from untreated, control siRNA-, or VEGF siRNA-transfected cells. Forty-eight hours later, whole-cell lysates were subjected to western blot analysis for VEGF. Incubation with an anti- $\beta$-actin antibody was used as a protein-loading control. (B) 
The effect of siRNA on mRNA expression in D-HCAECs. The efficiency of VEGF knockdown was calculated to be $80 \%$ by real-time quantitative RT-PCR. Data are expressed as mean \pm SEM $(\mathrm{n}=3),{ }^{* *} p$ $<0.01$. (C) VEGF secretion in response to in vitro treatment with $\operatorname{AGP}(1,3$, and $10 \mu \mathrm{M})$. HCAECs and D-HCAECs were treated with AGP for $24 \mathrm{~h}$, and VEGF secretion was assessed by performing ELISAs. Data from the VEGF assays are presented as mean \pm SEM $(n=3), * * p<0.01$. (D) VEGF-siRNAtransfected D-HCAEC cells were inhibited cell growth promotion. An equal number of cells $\left(1 \times 10^{4}\right.$ cells/well) were seeded in 96-well plates and cultured overnight at $37^{\circ} \mathrm{C}$ in an incubator with $5 \% \mathrm{CO}_{2}$. Subsequently, DMSO (vehicle), $10 \mu \mathrm{M}$ AGP (18:1), or $10 \mu \mathrm{M}$ cPA (18:1) were added to the medium, and the cells were incubated for $24 \mathrm{~h}$. The amount of orange formazan dye generated was determined by measuring the absorbance at $450 \mathrm{~nm}$ in a microplate reader. Data are expressed as mean \pm SEM $(\mathrm{n}=3)$, ${ }^{* *} p<0.01$. (E) Effect of cPA on mRNA expression and production of inflammatory cytokines To measure mRNA expression, D-HCAEC were cultured with $10 \mu \mathrm{M}$ cPA for $24 \mathrm{~h}$ in the presence or absence of $10 \mu \mathrm{M}$ AGP. mRNA levels of each gene were determined by quantitative real-time PCR. Data represent the mean \pm SE of triplicate independent experiments $\left({ }^{*} P<0.05\right.$ and ${ }^{* *} P<0.01$ vs. vehicletreated controls). To measure IL-6, and IL-8 concentrations in the culture media of D-HCAEC, After $24 \mathrm{~h}$ incubation, culture media were collected, and IL-6, and IL-8 concentrations were determined by ELISA. Data represent the mean \pm SE of triplicate independent experiments $\left({ }^{*} P<0.05\right.$ and ${ }^{* *} P<0.01$ vs. vehicle-treated controls).

Figure 8. Schematic representation of the proposed mechanism of cPA action in D-HCAECs. After treatment with AGP, cell growth and migration was induced in D-HCAECs, obtained from donors with type II diabetes. Moreover, addition of cPA suppressed the AGP-elicited growth and migration in DHCAECs. AGP increased VEGF secretion from the D-HCAECs, and this event was attenuated by cPA. These results suggest that cPA suppresses VEGF-stimulated growth and migration in D-HCAECs. 


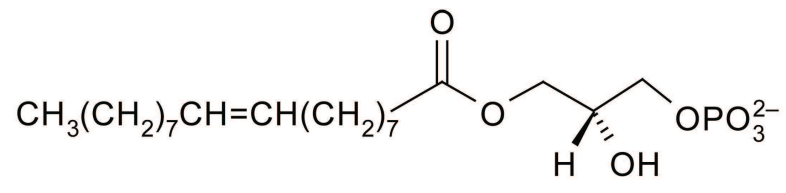

LPA 18:1

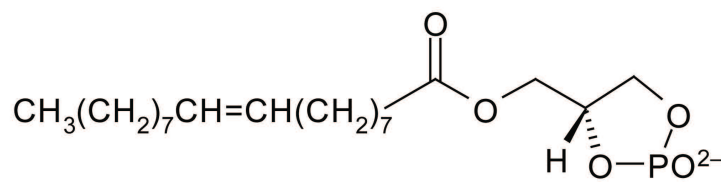

CPA 18:1

$\mathrm{CH}_{3}\left(\mathrm{CH}_{2}\right)_{7} \mathrm{CH}=\mathrm{CH}\left(\mathrm{CH}_{2}\right)_{8} \mathrm{O} \overbrace{\mathrm{H}=\mathrm{OH}} \mathrm{OPO}_{3}^{2-}$

AGP 18:1

Fig.1 
A

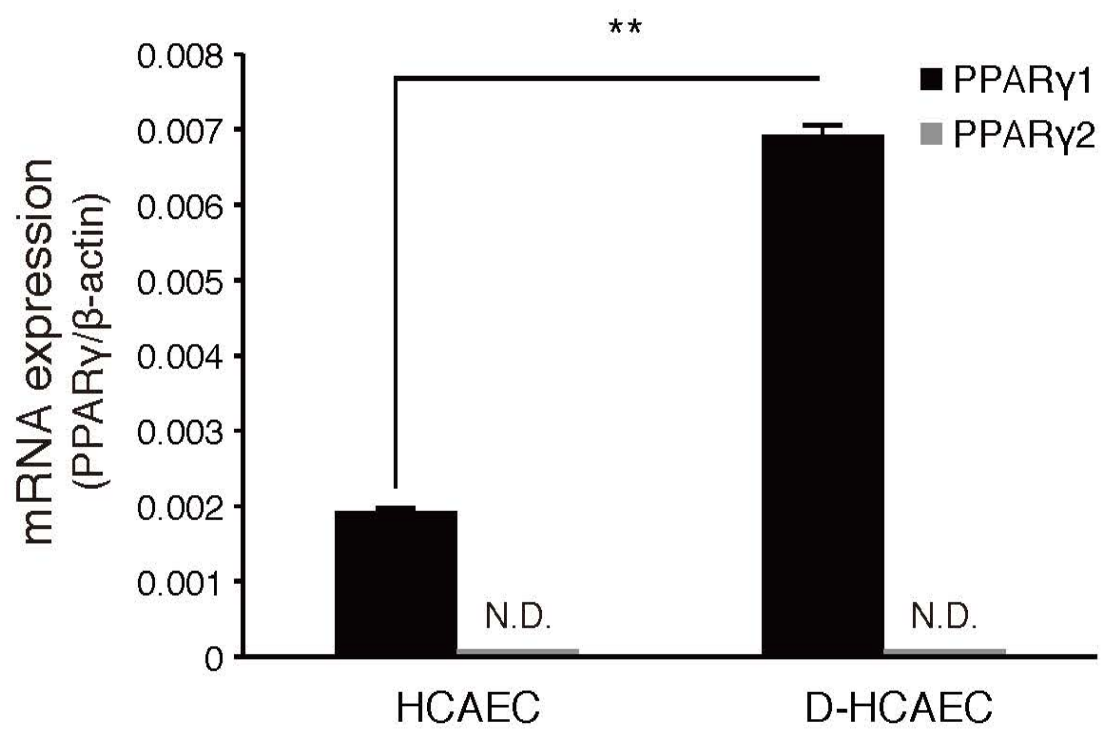

B

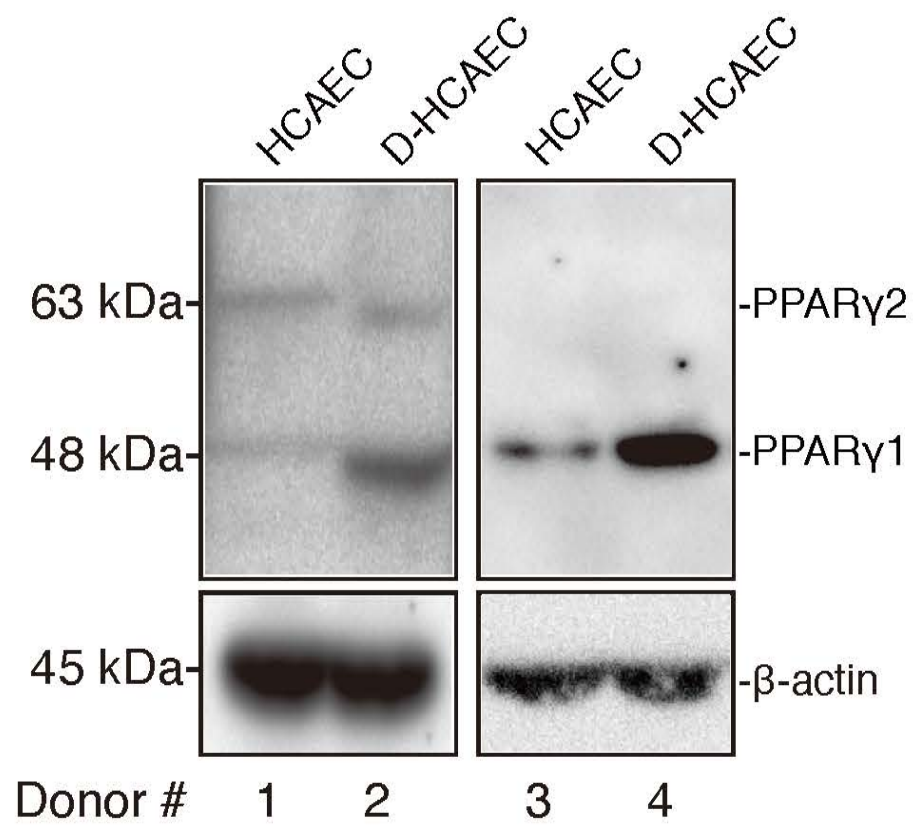

Fig.2 
A

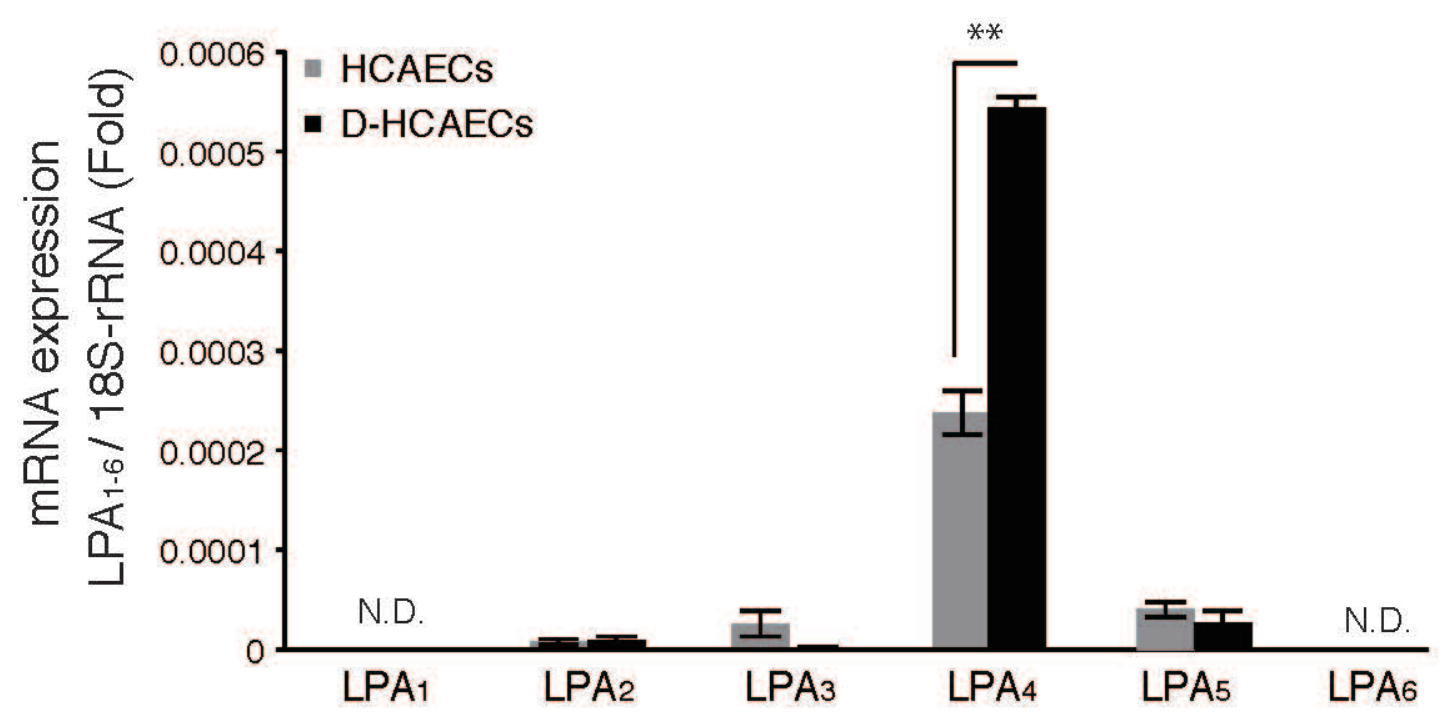

B
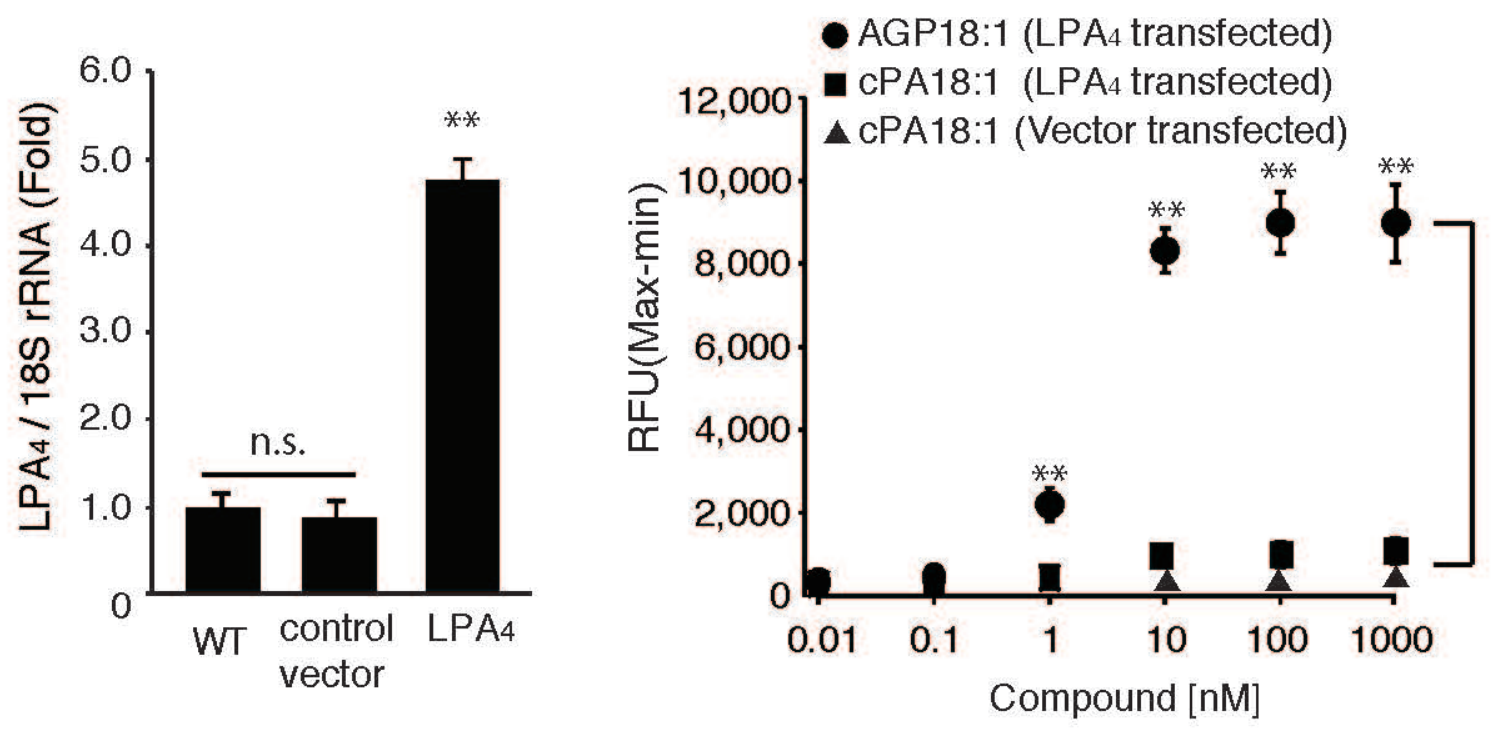

C
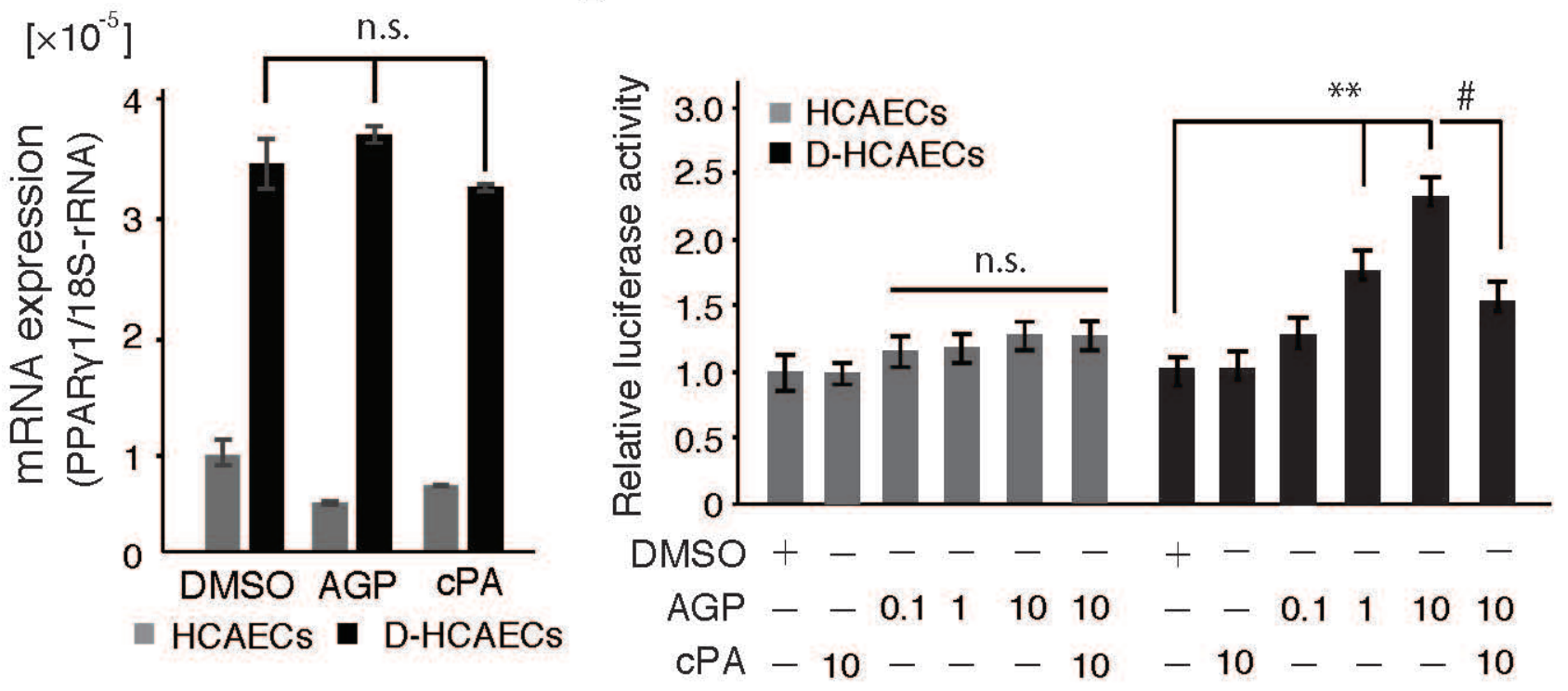

Fig.3 
A

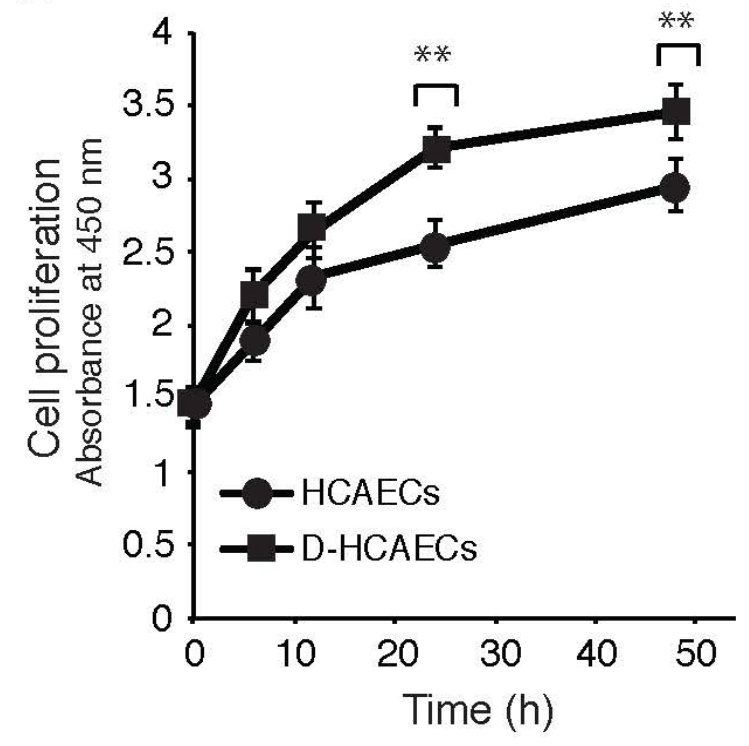

C

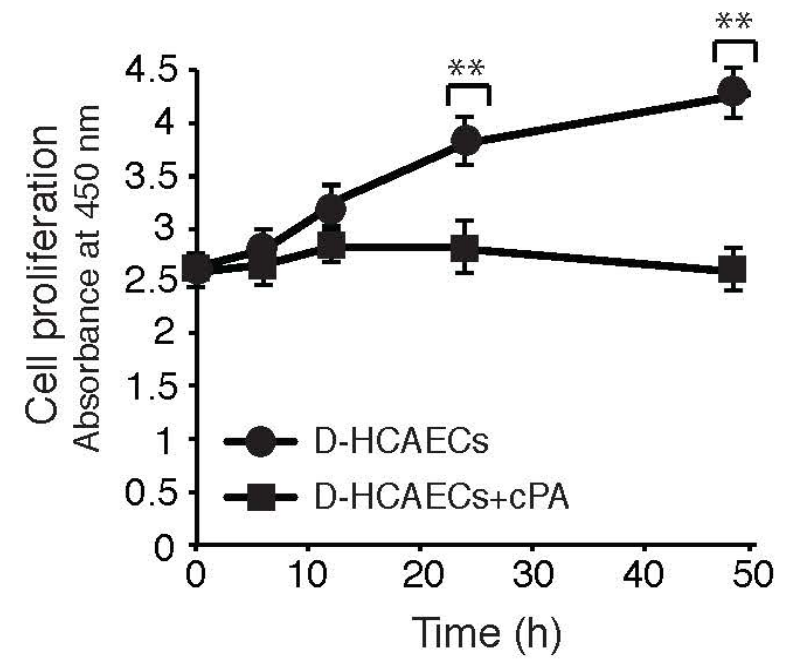

E

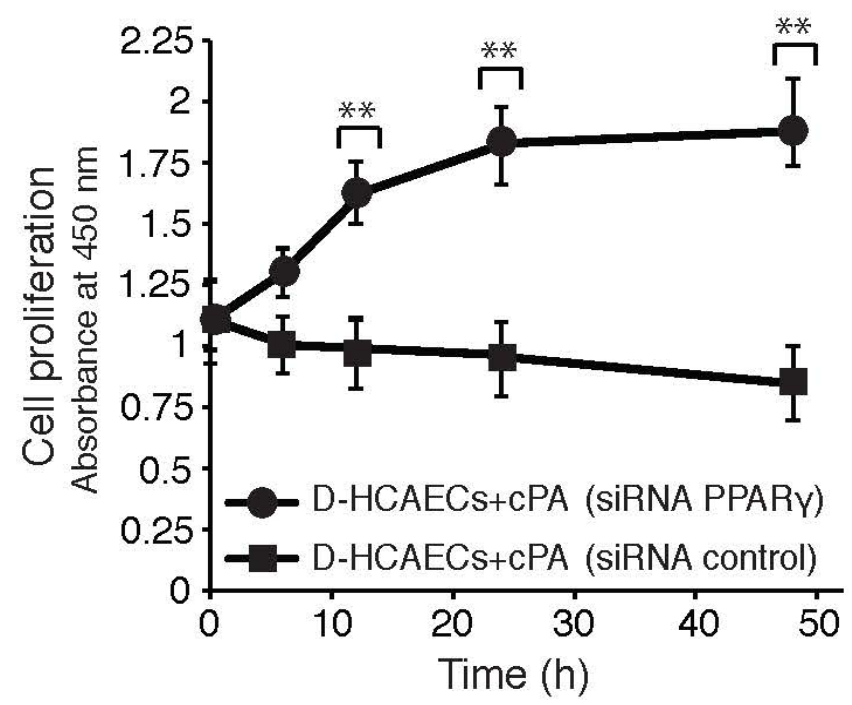

B

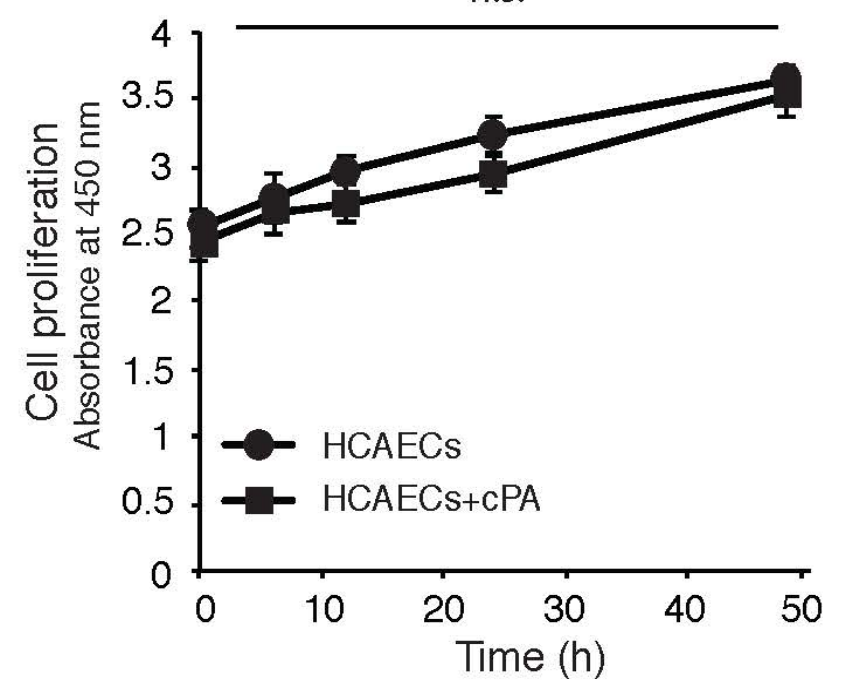

D

\section{D-HCAECs}

SIRNA SIRNA

Cont. PPARy1

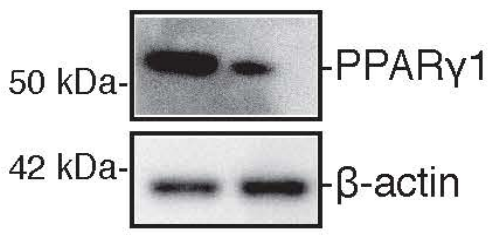

F

n.s.

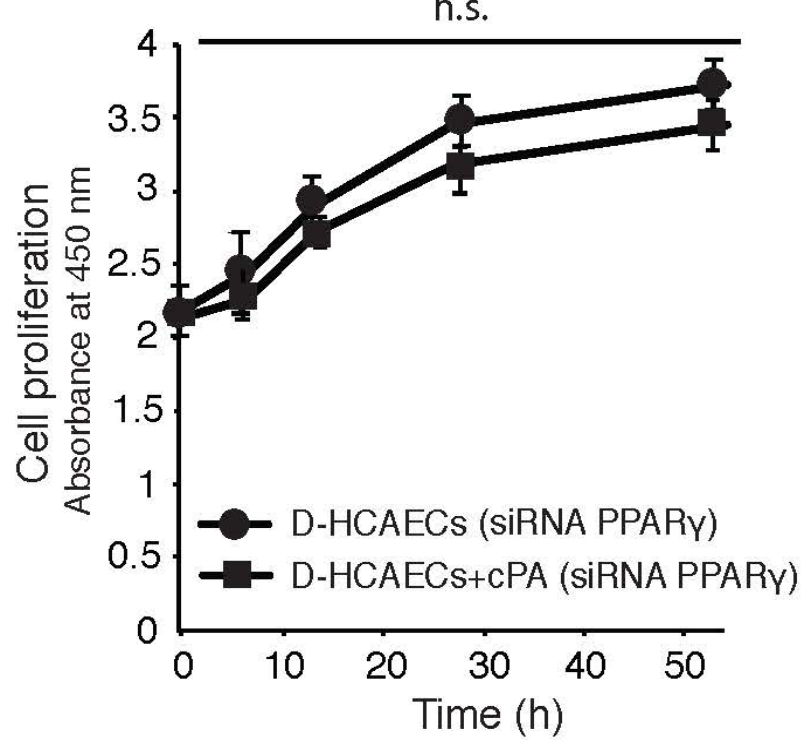

Fig.4 

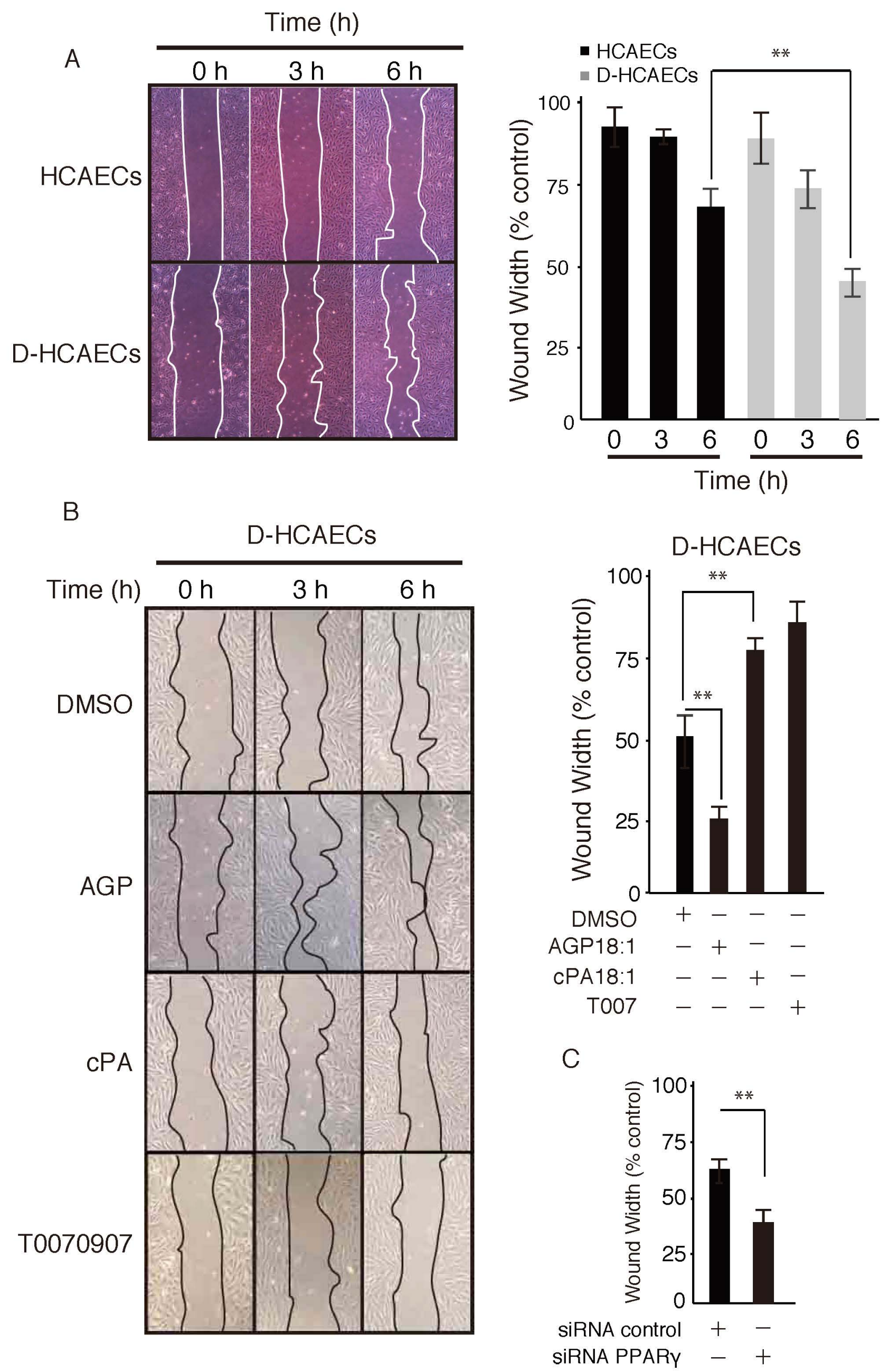

Fig.5 
A

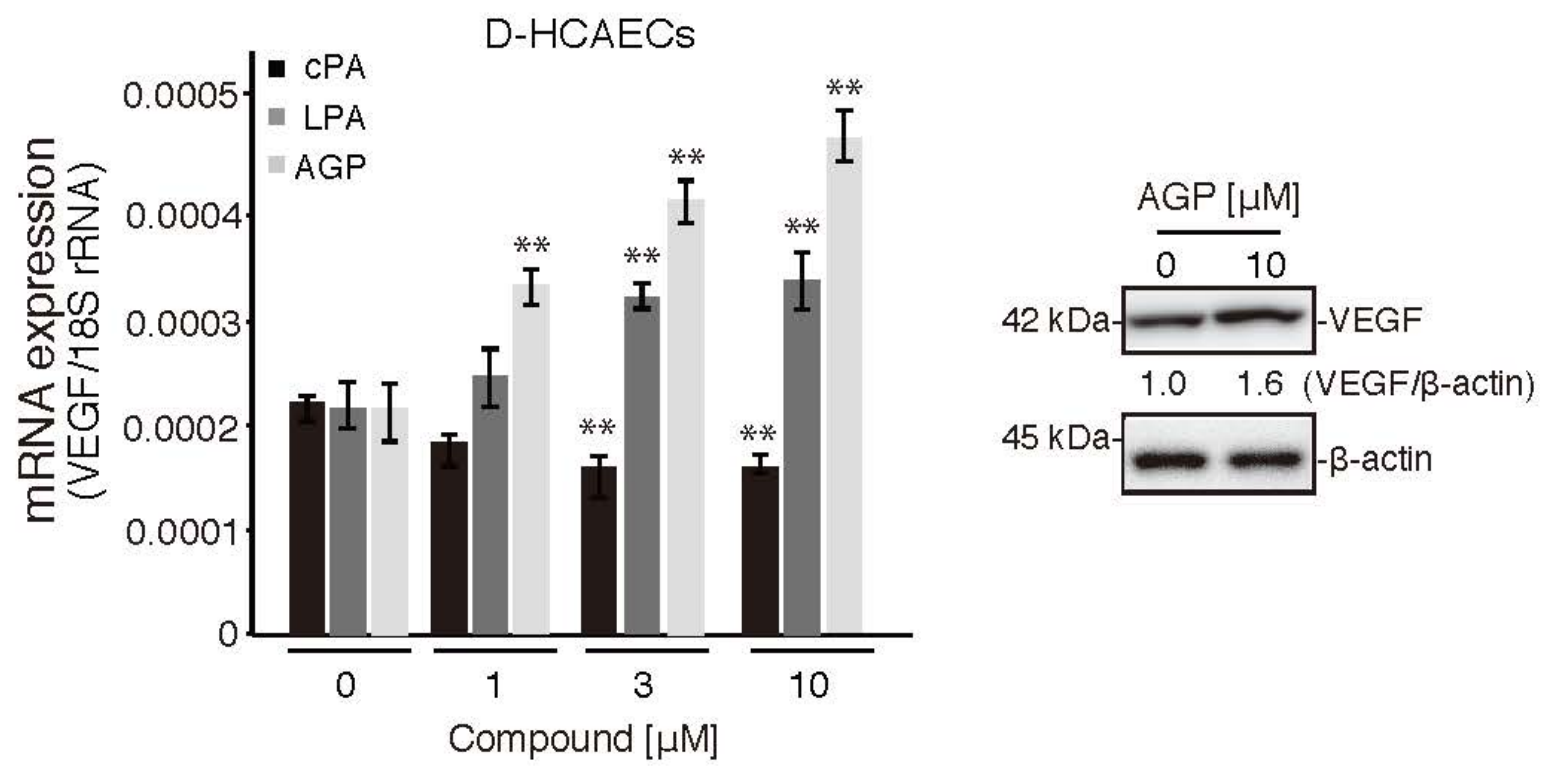

B

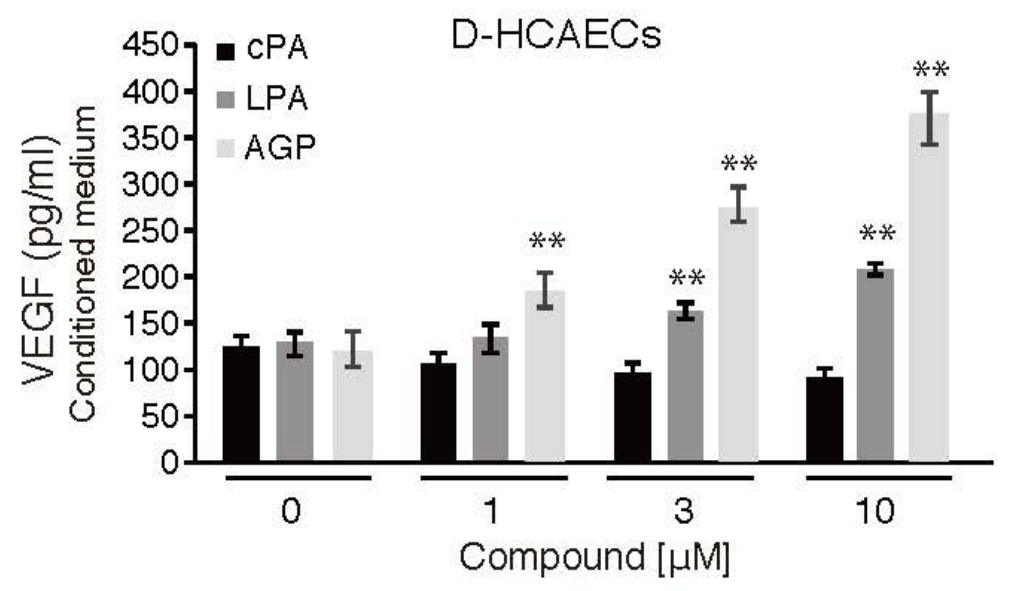

C

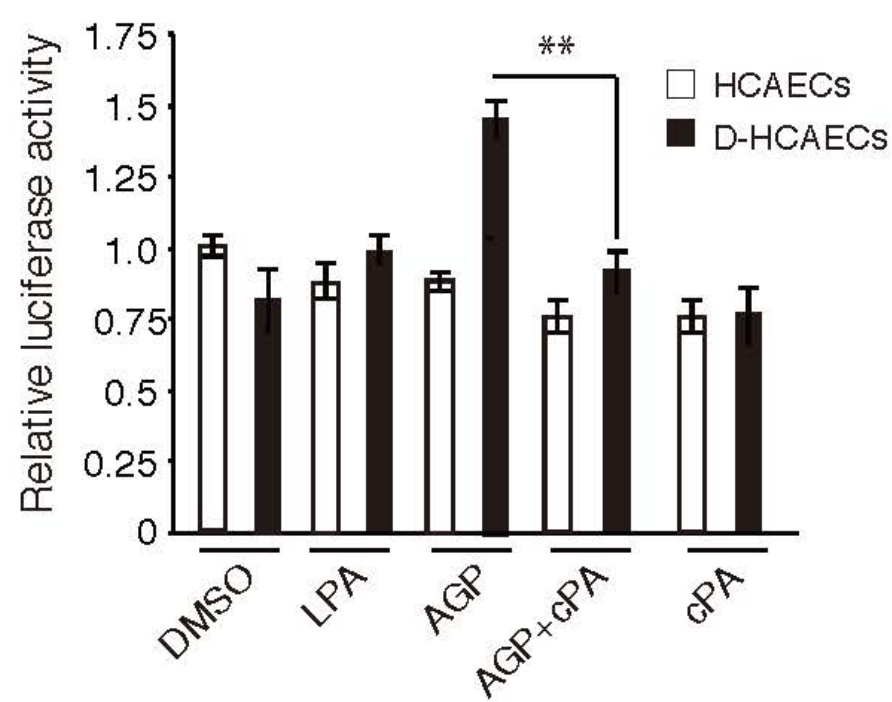

D

D-HCAECS

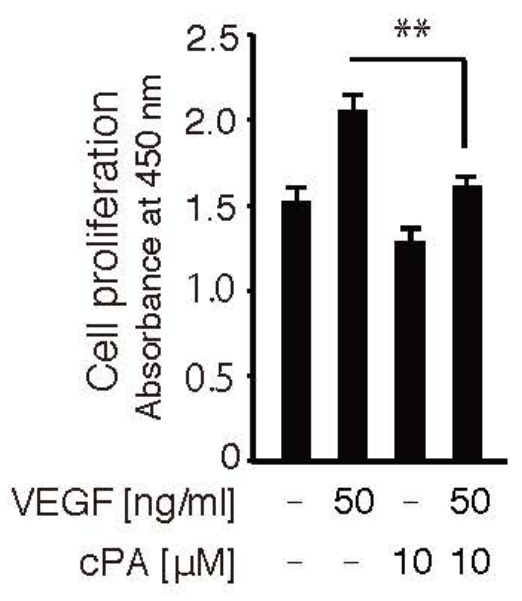

Fig. 6 
A

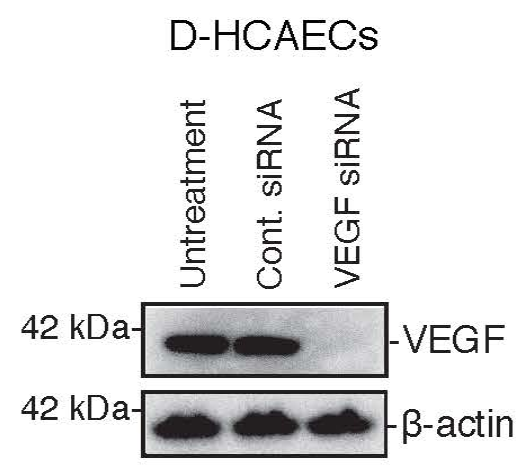

B

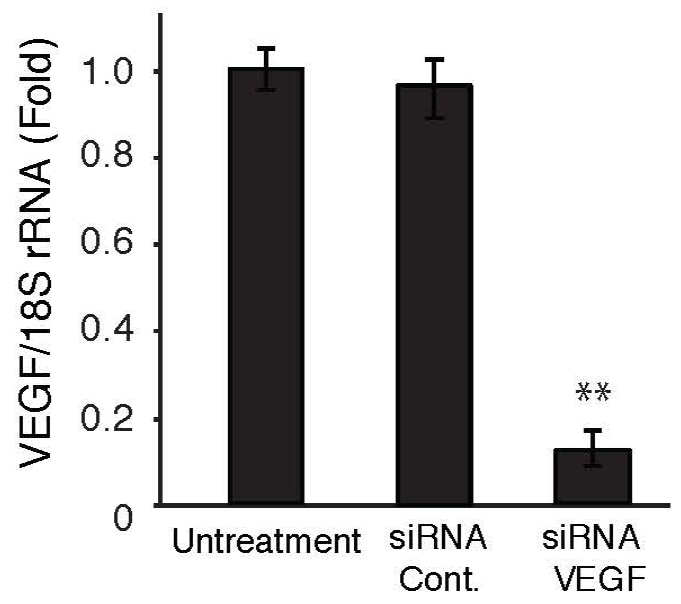

C

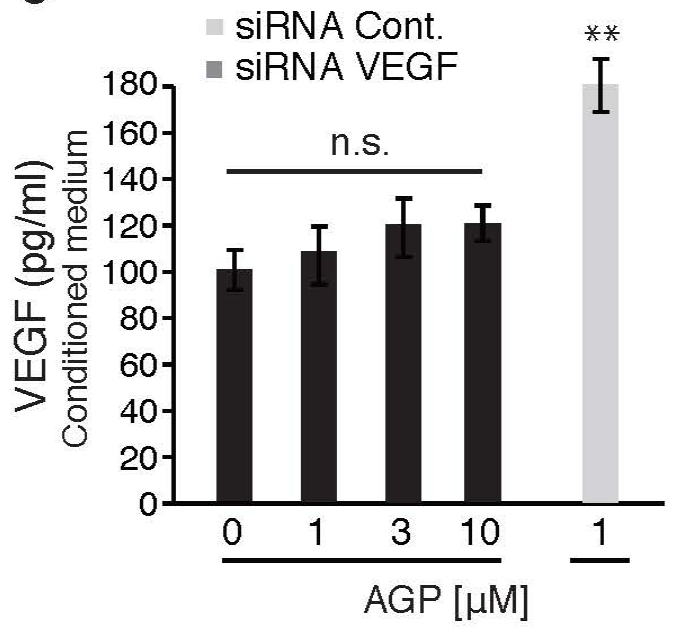

D

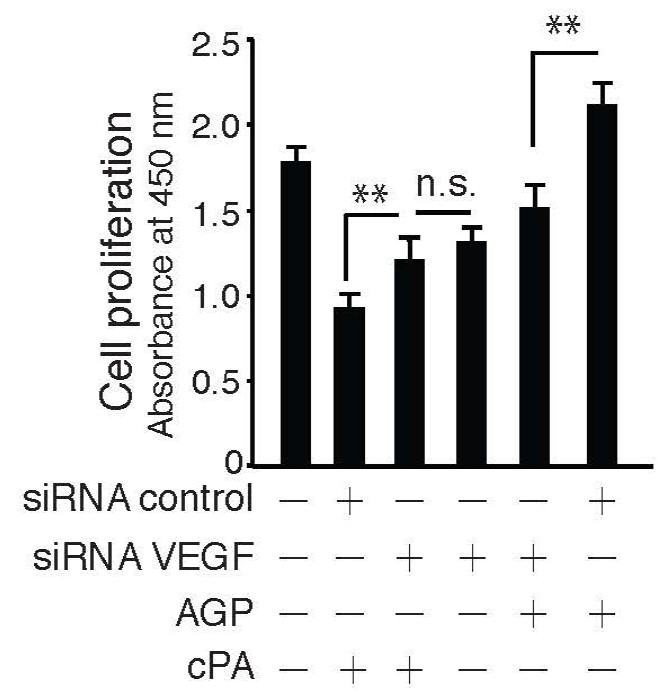

E

IL-6

IL-8

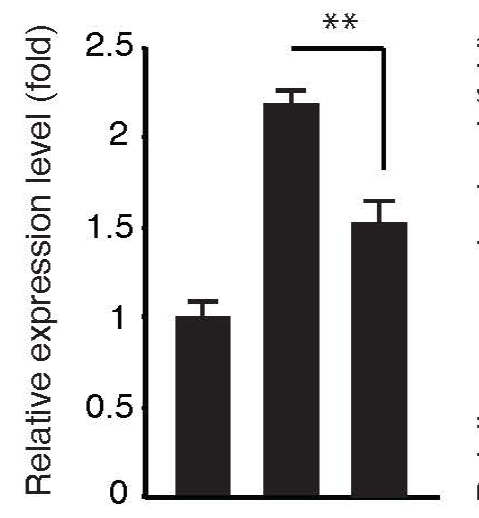

DMSO

AGP

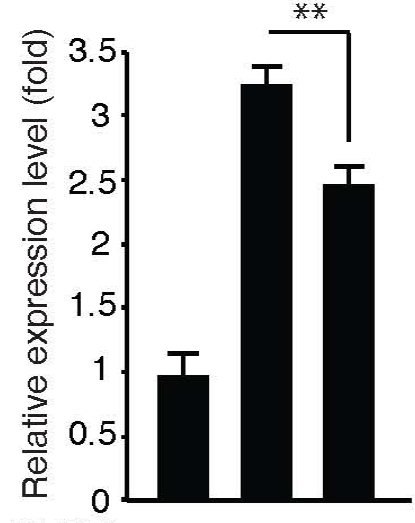

DMSO

AGP -++

$\mathrm{CPA}-\quad-+$

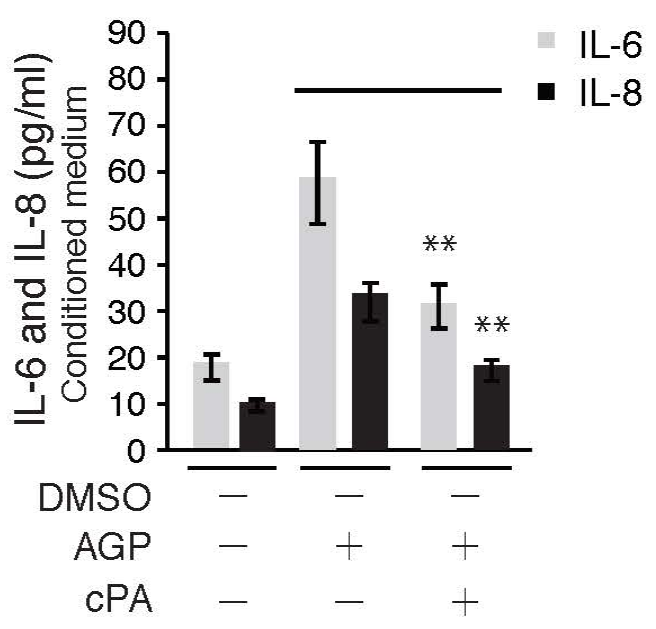

Fig.7 

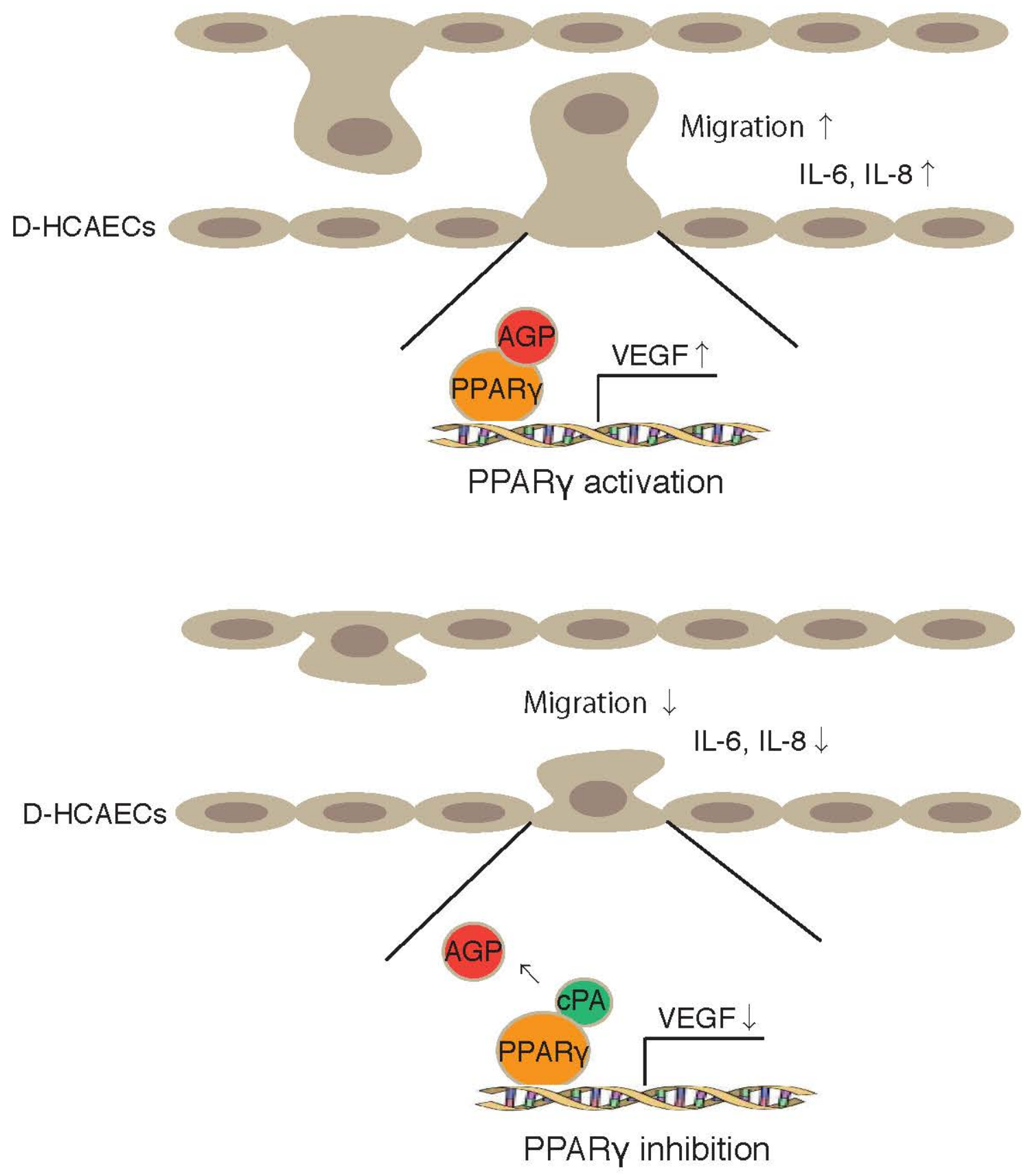

Fig. 8 